\title{
Improving Capacity in LMDS Networks Using Trellis-Coded Modulation
}

\author{
Ranjan Bose \\ Department of Electrical Engineering, Indian Institute of Technology (IIT) Delhi, Hauz Khas, New Delhi 110016, India \\ Email: rbose@ee.iitd.ac.in
}

Received 23 January 2004; Revised 5 July 2004; Recommended for Publication by Jonathon Chambers

\begin{abstract}
Local multipoint distribution systems (LMDS), which are operating worldwide in the frequency range of 26 to $43 \mathrm{GHz}$, have large bandwidths of 0.1 up to $2 \mathrm{GHz}$ but a limited area coverage of a few kilometers only. This is due to line-of-sight (LOS) constraints for reliable point-to-multipoint links as well as due to large propagation losses. The size of the macrocells illuminated by the base stations, where LOS exists, is approximately 1 to $5 \mathrm{~km}$. As suggested in the CRABS report (1999), the maximal spectral efficiency can be obtained with a dual frequency and polarization reuse plan. This frequency and polarization reuse leads to interference. In this paper, we report a new technique that uses trellis-coded modulation (TCM) for increasing the capacity of LMDS networks. Analytical expressions have been derived for pairwise error probability for both high and low SNR scenarios. Numerical simulations have shown that using the proposed TCM schemes in cochannel cells have resulted in a large decrease in interference, thereby allowing us to reduce the frequency reuse distance. In this paper, we present the strategy using two TCM schemes that allow the frequency reuse factor of unity, without compromising on the QoS caused by increased interference. Design rules for constructing TCM schemes are also proposed in this paper.
\end{abstract}

Keywords and phrases: broadband wireless access, trellis-coded modulation, cochannel interference, interference mitigation.

\section{INTRODUCTION}

Broadband radio access systems operating at millimeter waves, often called local multipoint distribution systems (LMDS), are currently being developed in the $26-43 \mathrm{GHz}$ frequency range, having bandwidths of 0.1 up to $2 \mathrm{GHz}$ $[1,2,3,4]$. LMDS are local cellular point-to-multipoint radio systems, delivering broadband services from a central transmitter or base station (BS) to fixed customer stations (CS) mounted on individual buildings, blocks of apartments, or buildings of residential as well as business customers within its cell size [5] (http://www.iec.org/online/tutorials/lmds/). These broadband radio access systems offer rapid infrastructure deployment and the ability to provide local content. They can be significantly cheaper to install than a cable system since only customers requesting LMDS are provided with terminals (extension on demand). As a result of the propagation characteristics of signals in this frequency range, LMDS systems use a cellular-like network architecture, though services provided are fixed, not mobile. In the United States, $1.3 \mathrm{MHz}$ of bandwidth $(27.5-28.35 \mathrm{GHz}$, 29.1-29.25 GHz, 31.075-31.225 GHz, 31-31.075 GHz, and $31.225-31.3 \mathrm{GHz}$ ) has been allocated for LMDS to deliver broadband services in a point-to-point or point-tomultipoint configuration to residential and commercial customers [5]. The $42 \mathrm{GHz}$ LMDS band proposed in Europe provides $2 \mathrm{GHz}$ of bandwidth. Rain attenuation and line of sight (LOS) play an important role at these frequencies [6, 7]. The capacity needed by each user has also increased over time as new services have been developed and this is likely to continue. The parameters for the traditional LMDS, suggested in the CRABS report (Telenor scheme) [8], uses two frequencies and two polarizations (vertical and horizontal) and cells which are essentially square.

In [9] the authors have used trellis-coded modulation (TCM) to reduce frequency reuse distance for the GSM network. In this paper, we propose to explore the possibility of using only a single frequency coupled with horizontal and vertical polarizations for the LMDS network. If we simply reduce the frequency reuse factor to unity, there is an increase in the cochannel interference (CCI). However, two TCM schemes $[10,11]$ are deployed in cochannel cells to reduce the CCI and thereby achieve similar performance as suggested in the Telenor scheme. The proposed scheme increases the capacity of the LMDS network without compromising on the quality of service (measured in BER), at the cost of increased complexity. The paper is organized as follows. Section 2 gives the description of the system under study. The basic concept of using TCM for increasing the capacity of the network is explained in Section 3. The performance analysis for the TCM schemes and the design criteria for low and high SNR scenarios are discussed in Section 4. 


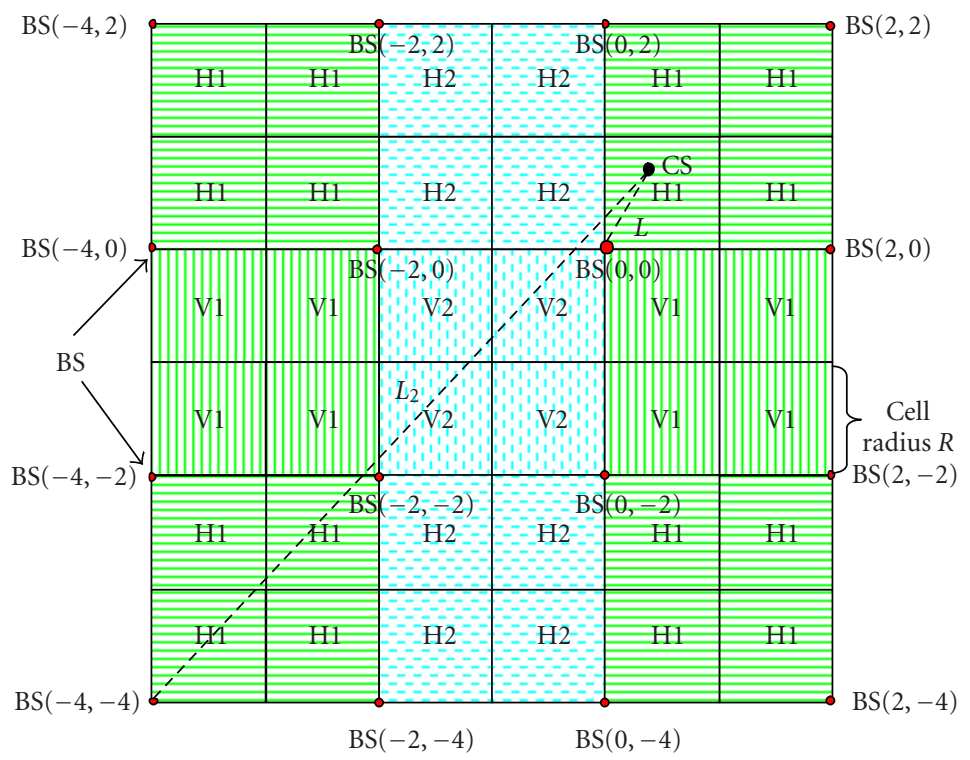

FIGURE 1: Dual frequency and polarization reuse plan as suggested in the Telenor scheme of the CRABS report [8]. The circles in the figure denote the position of the base stations, on which four $90^{\circ}$-sectoral horn antennas are mounted.

The specific TCM schemes are given in Section 5 and the results from numerical simulations are also presented. In Section 5 we also compare the proposed scheme to the existing one, and discuss the trade-offs. The paper concludes in Section 6.

\section{SYSTEM DESCRIPTION}

We have used the scheme suggested by Telenor in the CRABS report [8], as shown in Figure 1. The maximal spectral efficiency is obtained with a dual frequency and polarization reuse plan as shown, where the macrocells are square in shape. For this architecture, the cochannel cells appear in the fifth tier. "H1" implies that the cell uses horizontal polarization, frequency 1, V1 implies vertical polarization, frequency 2, and so forth. The locations of the base stations are marked by circles. The half-power beamwidth (HPBW) of the transmitter at the BS is $90^{\circ}$, thus leading to cells that are square in shape. The receivers are usually parabolic dish antennas mounted on the rooftop of the customers with a narrow $\mathrm{HPBW}$ of $2^{\circ}-5^{\circ}$. In the figure, $\mathrm{BS}(0,0)$ is the desired base station for the CS. The interfering BS is BS $(-4,-4)$. The customer antenna, though looking at its own $\mathrm{BS}(0,0)$, picks up interference from BS $(-4,-4)$.

The LOS interference calculations are done based on a $\mathrm{H}$-plane or E-plane sectoral horn antennas for the transmitter at the base stations, providing horizontal and vertical polarization, respectively. The transmitter antennas have a HPBW approximately equal to $90^{\circ}$. The receiver antennas of the subscribers are assumed to have a circular aperture with a parabolic taper on pedestal with a $10 \mathrm{~dB}$ edge illumination, having high gain between $30-40 \mathrm{~dB}$ with a very narrow $\mathrm{HPBW}$ of $2^{\circ}-5^{\circ}$ for a diameter of about 10 to $24 \mathrm{~cm}$.
The carrier-to-cochannel interference $(\mathrm{C} / \mathrm{I})$ ratio has been calculated for LOS under clear weather conditions without any power control strategy using

$$
\begin{aligned}
\mathrm{C} / \mathrm{I}\left(d_{1},\right. & \left.d_{2}, p\right) \\
= & {\left[\operatorname{EIRP}_{D}-\operatorname{EIRP}_{I}\right]+\left[G_{R}(0)-G_{R}(\theta)\right] } \\
& -\left[L_{\mathrm{fs}}\left(d_{1}\right)-L_{\mathrm{fs}}\left(d_{2}\right)\right]-\left[A_{\mathrm{cs}}\left(d_{1}\right)-A_{\mathrm{cs}}\left(d^{2}\right)\right] \\
& -A_{f}\left(d_{2}, p\right)(\mathrm{dB}),
\end{aligned}
$$

where $\operatorname{EIRP}_{D}$ and $\operatorname{EIRP}_{I}$ are the equivalent isotropic radiated powers of the desired and interfered signals at the customer location, $G_{R}(\theta)$ is the receiver antenna gain at an angle $\theta$ off the boresight, $L_{\mathrm{fs}}$ is the free space path loss, $A_{\mathrm{cs}}$ is the attenuation during clear sky, $A_{f}$ is the short-term enhancement due to atmospheric multipath and focussing effects, $p$ is the time percentage for which $A_{f}$ exceeds a certain value $[12,13], d_{1}$ and $d_{2}$ are the distances in $\mathrm{km}$ from the $\mathrm{BS}$ of the desired and cochannel cell, respectively. At $42 \mathrm{GHz}, A_{\mathrm{cs}}$ has been taken as $0.2 \mathrm{~dB} / \mathrm{km}$ [14]. The short-term enhancement due to atmospheric multipath and focussing effects, $A_{f}$, is determined using the formula [12]

$$
A_{f}=2.6\left(1-e^{-d_{2} / 10}\right) \log _{10} \frac{p}{50}(\mathrm{~km}) .
$$

\section{THE PROPOSED IDEA}

We propose to use only a single frequency, two polarizations (horizontal and vertical), and two TCM schemes as opposed to the existing two frequency and two polarizations (2F2P). If we use only one frequency and two polarizations $(1 \mathrm{~F} 2 \mathrm{P})$, the frequency reuse distance decreases, resulting in higher levels of cochannel and adjacent channel interference. To overcome the effect of smaller reuse distance, we deploy two different TCM schemes in the cochannel cells as depicted in Figure 2. 


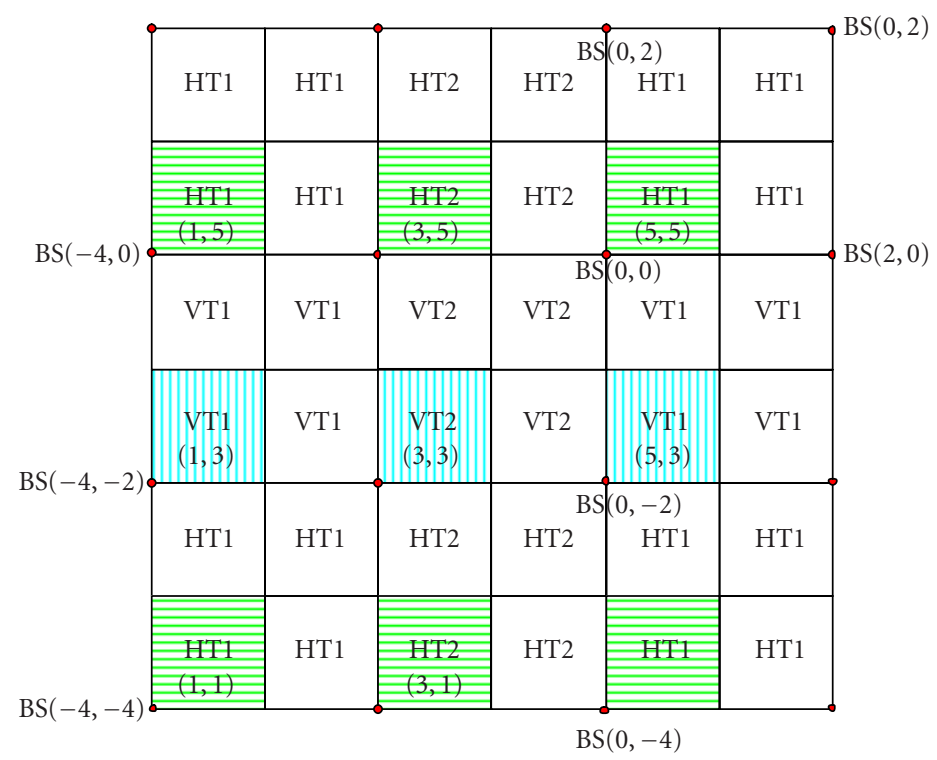

Figure 2: Frequency, polarization, and TCM scheme planning (1F2P2T).

Note that in this figure we are using one coordinate scheme for BS and another one to label the cells. These TCM schemes are so designed that the sequence of symbols generated by one trellis is rejected by the other. As a result of the coding gain, the effective CCI will be reduced.

As is clear from Figure 2, we have only one frequency and two polarizations. Consider cell $(5,5)$. It will get CCI from all " $\mathrm{H}$ " cells, that is, cell using the horizontal polarization. The closest cells using horizontal polarization are $(1,5),(3,5)$, $(3,1),(5,1)$, and $(1,1)$. In the Telenor scheme, the cochannel cells of the first tier were only $(1,5),(5,1)$, and $(1,1)$. Considering a receiver antenna beamwidth of $5^{\circ}$ at $42 \mathrm{GHz}$, the different areas in the cell experiencing interference are given in Table $1[15,16,17]$.

Figure 3a shows the interference levels within the cell for the Telenor scheme (2F2P). The figure is color coded. The regions depicted in light shades of gray represent $\mathrm{C} / \mathrm{I}$ above $30 \mathrm{~dB}$ while the regions in dark shades of gray represent high interference levels. Due to the directional receiver antenna at the customer location, we find the high interference regions in the cell appearing as wedges. The figure shows that certain areas within the cell are bad in terms of the CCI. Since this is a fixed wireless communication scenario, these interferences affected regions will remain so. Figure $3 \mathrm{~b}$ depicts the scenario where the frequency reuse distance has been reduced. As expected, the reduction of two frequencies to one, that is, $(2 \mathrm{~F} 2 \mathrm{P})$ to $(1 \mathrm{~F} 2 \mathrm{P})$, has resulted in higher interference levels, leading to an increase in the areas within the cell with low $\mathrm{C} / \mathrm{I}$. We observe a drastic increase in the percentage of cell area experiencing interference, as shown by the figures and Table 1.

For the sake of illustration, we say that the satisfactory performance requires the symbol error rate, $P_{\text {sym }} \approx 10^{-5}$. Suppose the modulation scheme to be used is 8-PSK. If gray coding is used to assign codewords to the symbols, the bit er- ror rate, $P_{e} \approx P_{\text {sym }} / \log _{2}(M)$, where $M=8$ for 8 -PSK. So, 8 PSK cannot be used in those regions within the cell where the $\mathrm{C} / \mathrm{I}<10 \mathrm{~dB}$ [18]. This implies that for the Telenor scheme, approximately $5.48 \%$ of the area of the cell will have unsatisfactory quality of service (QoS) (see Table 1). On the other hand, for the single-frequency, two-polarization scheme, we have more than $10 \%$ area with unsatisfactory QoS (i.e., C/I $<10 \mathrm{~dB})$. To mitigate the higher interference level, we would use TCM in combination with 8-PSK. We will call this 8PSK/TCM. It will be shown in Section 5 that this scheme lowers the $P_{\text {sym }}$, thus reducing the areas within the cell with poor QoS. Besides, this strategy requires less bandwidth without compromising on the QoS.

The basic objective is to improve the capacity of the system by reducing the frequency reuse distance. In the traditional LMDS frequency-polarization reuse scheme [8], two frequency bands and two polarizations are used. So, effectively they have four colors $(\mathrm{H} 1, \mathrm{H} 2, \mathrm{~V} 1, \mathrm{~V} 2)$ to color the cells and reduce the CCI (Figure 1). In our proposed technique we use only one frequency band, two polarizations, and two TCM schemes. Effectively, we also have four colors (HT1, HT2, VT1, VT2) to color the cells in order to reduce interference. So, even though the reuse distance has been decreased (by discarding a frequency band), due to the deployment of different TCM schemes in the cochannel cells, the interference is mitigated. This brings us to the question of how such TCM schemes should be designed. Intuitively, the signals encoded with TCM scheme 1 should be rejected by the TCM decoder 2 and vice versa. If this happens, then we will truly be able to reduce interference from cochannel cells. Since we want one coding scheme to reject the signals coded by the other scheme, we can draw a rough parallel with CDMA. This is a very loose comparison. There is no concept of "orthogonality" of two TCM schemes as is present in CDMA (though the initial concept of orthogonal TCM design has 


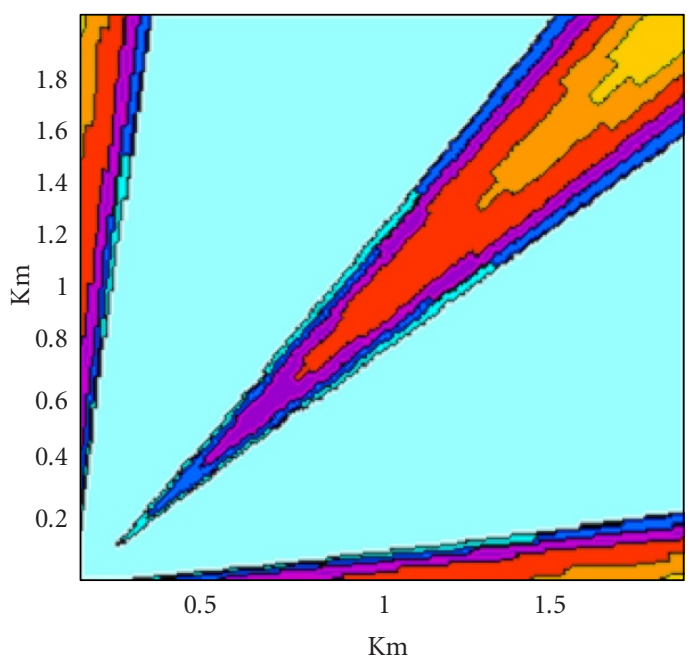

(a)

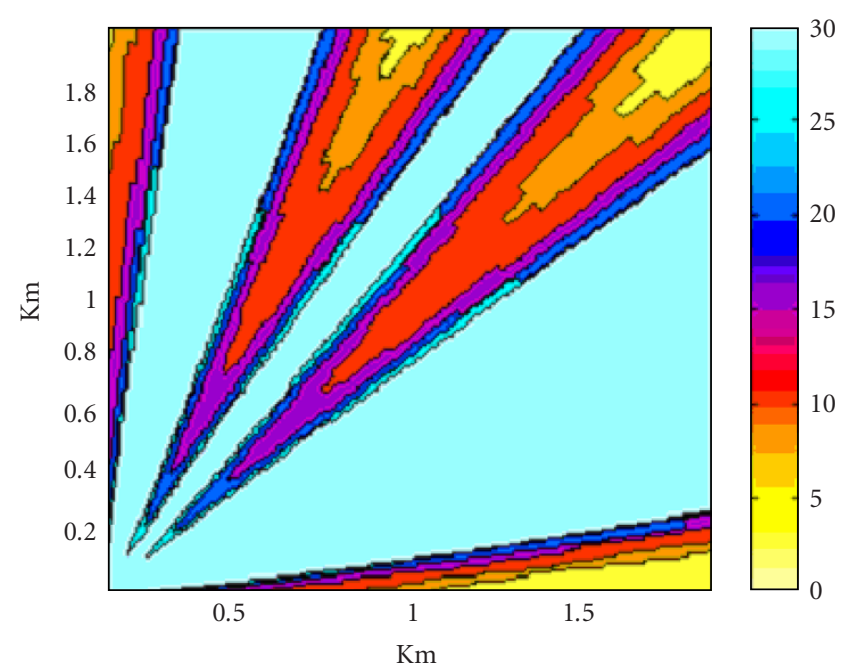

(b)

Figure 3: Color-coded areas within the cell that experience C/I levels below a specified level for CCI. The units of the color bar is in dB. (a) 2 frequencies, 2 polarizations $(2 \mathrm{~F} 2 \mathrm{P})$; (b) 1 frequency, 2 polarizations $(1 \mathrm{~F} 2 \mathrm{P})$.

TABLE 1: Areas within the cell that experience different levels of CCI.

\begin{tabular}{l|cc}
\hline C/I value & \multicolumn{2}{|c}{ Area within the cell (\%) } \\
\hline Below 8 dB & Telenor scheme (2F2P) & 1 frequency, 2 polarization \\
Below 10 dB & 1.37 & 3.77 \\
Below 15 dB & 5.48 & 10.10 \\
Below 20 dB & 15.14 & 24.09 \\
Below 25 dB & 22.24 & 34.07 \\
Below 30 dB & 27.33 & 41.76 \\
Above 30 dB & 29.87 & 45.04 \\
\hline
\end{tabular}

been explored in [9]). The best we can do is to design the two TCM schemes in such a manner that they mutually reject each other's signals (in the same way as noise is rejected). It should be noted here that we do need two TCM schemes. From Figure 2 it is clear that four colors (HT1, HT2, VT1, VT2) are needed for the polarization-code planning. If we just use one TCM scheme, we will not be able to reject the CCI in all the cochannel cells.

\section{DESIGN CRITERIA FOR TCM FOR LMDS}

In this section we develop the design rules for the construction of TCM schemes for the LMDS architecture. As depicted in Figure 1, the CS receives signal both from the desired BS as well as the interfering BS. Consider the received signal at a certain CS

$$
r(t)=a(t) s(t)+b(t) u(t)+n(t), \quad 0 \leq t \leq T,
$$

where $a(t)$ is the attenuation for the desired signal, $b(t)$ is the attenuation for the interfering signal, $n(t)$ is the additive white Gaussian noise with a two-sided spectral density of $N_{0} / 2, s(t)=\sum_{n=0}^{N-1} s_{n} \phi_{n}(t)$ are the symbols of the MPSK in the $N$-dimensional signal space generated by the desired BS, and $u(t)=\sum_{n=0}^{N-1} u_{n} \phi_{n}(t)$ are the symbols generated by the interfering BS. Here, $\phi_{n}(t), n=0,1, \ldots, N-1$, represent the $N$ orthonormal functions and $s_{n}$ and $u_{n}$ are the projections of $s(t)$ and $u(t)$ onto the function $\phi_{n}(t)$, respectively, that is, $s_{n}=\int_{0}^{T} s(t) \phi_{n}^{*}(t) d t$ and $u_{n}=\int_{0}^{T} u(t) \phi_{n}^{*}(t) d t$.

Since this is a fixed broadband wireless access scenario, both $a(t)$ and $b(t)$ can be assumed to have a constant envelope and phase over the interval $0 \leq t \leq T$. Hence (3) can be expressed as

$$
r(t)=a s(t)+b u(t)+n(t), \quad 0 \leq t \leq T .
$$

We assume that the receiver performs coherent detection [10]. For the coherent demodulator block (Figure 4), the integrator outputs, that is,

$$
r_{j}=\int_{0}^{T} r(t) \phi_{j}^{*}(t) d t, \quad j=0,1, \ldots, N-1,
$$




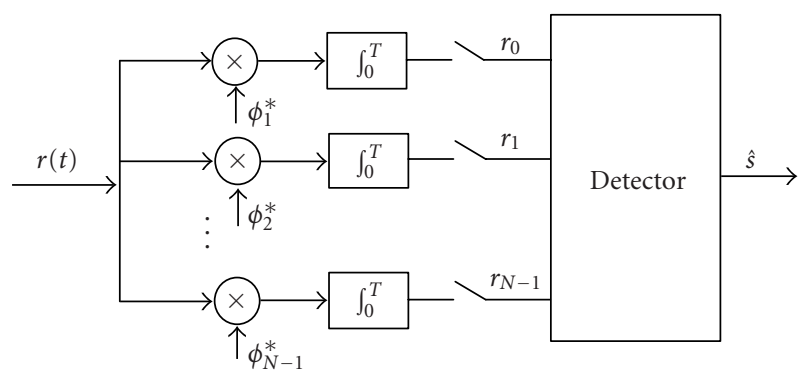

Figure 4: Block diagram of the coherent demodulator.

constitute the components of a random vector $\mathbf{r}=\left(r_{0}\right.$, $\left.r_{1}, \ldots, r_{N-1}\right)$. For the given (fixed) receiver location, from (4) we have

$$
\mathbf{r}=a \mathbf{s}+b \mathbf{u}+\mathbf{n},
$$

where $\mathbf{s}=\left(s_{0}, s_{1}, \ldots, s_{N-1}\right), \mathbf{u}=\left(u_{0}, u_{1}, \ldots, u_{N-1}\right), \mathbf{n}=$ $\left(n_{0}, n_{1}, \ldots, n_{N-1}\right)$, and $n_{j}=\int_{0}^{T} n(t) \phi_{j}^{*}(t) d t$.

Assuming that $n(t)$, hence $\mathbf{n}$, is statistically independent of $\mathbf{s}$ and $\mathbf{u}$, it can be seen from (6) that $\mathbf{r}$ is a random vector whose components $\left\{r_{j}\right\}$ are statistically independent Gaussian random variables with mean $E\left[r_{j}\right]=a s_{j}+b u_{j}$ and variance $N_{0} / 2$. Hence, we can write the conditional density of $\mathbf{r}$, given the attenuations $a$ and $b$, and the transmitted signal $s$ and $u$, as follows:

$$
p_{N}(\mathbf{r} \mid a, b, s, u)=\prod_{j=0}^{N-1} \frac{1}{\sqrt{\pi N_{0}}} e^{-\left|r_{j}-a s_{j}-b u_{j}\right|^{2} / N_{0}} .
$$

We have assumed here that channel state information is available. This is a fair assumption as this is a fixed broadband wireless access scenario and it is easy to measure the channel parameters.

Next, consider the discrete time model for the system as shown in Figure 5. Here, we use TCM 1 to encode the bit stream from the desired BS. The decoding is done using the Viterbi decoding where a sequence of symbols is taken for the purpose of decoding. Let the input bits be encoded by the TCM 1 to produce a sequence of signals $\mathbf{s}_{l}=\left(s_{1}, s_{2}, \ldots, s_{l}\right)$, where each signal $s_{k}$ is a two-dimensional vector chosen from an MPSK signal set. Here the subscript " $k$ " denotes the symbols in different time instances. Similarly, let $\mathbf{u}_{l}=$ $\left(u_{1}, u_{2}, \ldots, u_{l}\right)$ represent the sequence of signals generated in the interfering cell, where each signal $u_{k}$ is also a twodimensional vector chosen from the same MPSK signal set. The cell under consideration and the interfering cell differ in the TCM schemes deployed in the respective cells. Since the receiver performs coherent detection, the received signal at time $k$ can be written as

$$
r_{k}=a s_{k}+b u_{k}+n_{k}
$$

where $a$ is the attenuation for the desired signal, $b$ is the attenuation for the interfering signal, and $n_{k}$ is the sample of a zero-mean complex Gaussian noise process. For a given cell,

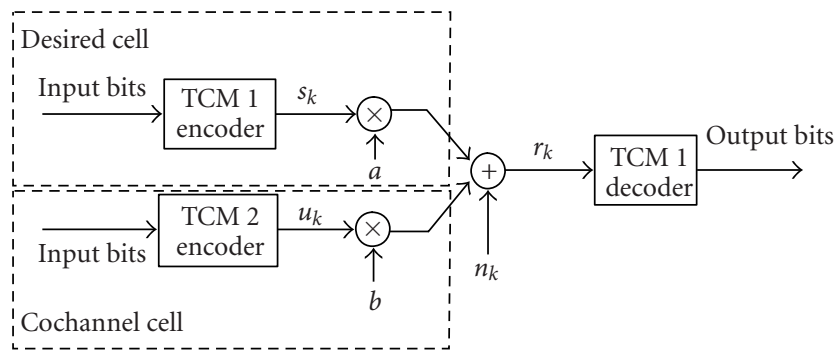

Figure 5: The baseband discrete time model for the system.

both $a$ and $b$ are random variables, the values of which depend on the location of the receiver (CS) inside the cell. We now calculate the pairwise error probability $P_{2}\left(\mathbf{s}_{l}, \hat{\mathbf{s}}_{l}\right)[10]$. As mentioned before, we assume that a maximum likely decoder is being used (Viterbi decoding) and the channel state information is available. The metric related to the conditional channel probabilities is given by $m\left(\mathbf{r}_{l}, \mathbf{s}_{l}\right)=\ln p_{N}\left(\mathbf{r}_{l} \mid \mathbf{s}_{l}, \mathbf{u}_{l}\right)$ [11]. Using (7), the decoding metric can be written as

$$
m\left(r_{k}, s_{k} ; a, b\right)=-\left|r_{k}-a s_{k}-b u_{k}\right|^{2},
$$

where the variance factor $N_{0} / 2$ is discarded since it is independent of the transmitted signal and has no bearings on the decoder decision.

The pairwise error probability is given by

$$
P_{2}\left(\mathbf{s}_{l}, \hat{\mathbf{s}}_{l} \mid a, b\right)=\operatorname{Pr}\left[m\left(\mathbf{r}_{l}, \hat{\mathbf{s}}_{l} ; a, b\right) \geq m\left(\mathbf{r}_{l}, \mathbf{s}_{l} ; a, b\right) \mid a, b\right] .
$$

The conditional pairwise error probability can be upperbounded by using the Chernoff bound as

$$
\begin{aligned}
& P_{2}\left(\mathbf{s}_{l}, \hat{\mathbf{s}}_{l} \mid a, b\right) \leq E_{\mathbf{n}}\left[\exp \left(\eta\left\{m\left(\mathbf{r}_{l}, \hat{\mathbf{s}}_{l} ; a, b\right)-m\left(\mathbf{r}_{l}, \mathbf{s}_{l} ; a, b\right)\right\}\right) \mid a, b\right] \\
&=\prod_{k=1}^{l} E_{n_{k}}\left[\operatorname { e x p } \left(\eta \left\{\left|r_{k}-a s_{k}-b u_{k}\right|^{2}\right.\right.\right. \\
&\left.\left.\left.-\left|r_{k}-a \hat{s}_{k}-b u_{k}\right|^{2}\right\}\right)\right],
\end{aligned}
$$

where $\eta$ is the Chernoff parameter. From (8) and (11) we can write

$$
\begin{array}{r}
\prod_{k=1}^{l} E_{n_{k}}\left[\exp \left(\eta\left\{\left|r_{k}-a s_{k}-b u_{k}\right|^{2}-\left|r_{k}-a \hat{s}_{k}-b u_{k}\right|^{2}\right\}\right)\right] \\
=\prod_{k=1}^{l} \exp \left(-\eta a^{2}\left(1-N_{0} \eta\right)\left|s_{k}-\hat{s}_{k}\right|^{2}\right) .
\end{array}
$$

This upper bound can be tightened by optimizing the Chernoff parameter $\eta$. The optimal value of $\eta$ is found out to be $\eta_{\text {opt }}=1 / 2 N_{0}$. Thus we have

$$
P_{2}\left(\mathbf{s}_{l}, \hat{s}_{l} \mid a, b\right) \leq \prod_{k=1}^{l} \exp \left(-\frac{a^{2}}{4 N_{0}}\left|s_{k}-\hat{s}_{k}\right|^{2}\right) .
$$


We observe that the right-hand side of (13) is independent of $b$. Intuitively, this can be explained as follows. Viterbi decoding of a TCM scheme chooses the most likely path in the trellis, given the received signal. An error occurs when an incorrect path is decoded as the most likely path instead of the correct path in the trellis. The presence of the interfering signal equally hampers the decision process for the correct path (corresponding to $\mathbf{s}_{l}$ ) as well as the wrongly decoded path (corresponding to $\hat{\mathbf{s}}_{l}$ ). Thus the pairwise error probability (the probability of choosing $\hat{\mathbf{s}}_{l}$ over $\mathbf{s}_{l}$ ) is independent of $b$.

To determine the pairwise probability for the entire cell, we need to average over the probability density function (pdf) of $a$, which is calculated as follows.

\section{Probability density function of $\mathbf{a}_{l}$}

We approximate the region of interest by a quarter within the square LMDS cell as shown in Figure 6. $R_{\min }$ is the minimum distance from the desired BS where a customer site can be located and $R$ is the length of the side of the square cell (cell radius). The received power at the customer site can be written as [19]

$$
P_{R}=\frac{P_{T} G_{T} G_{R} A_{\mathrm{cs}} \lambda^{2}}{\left(4 \pi^{2}\right)^{2} L d^{2}}
$$

where $P_{T}$ is the transmit power, $G_{T}$ is the transmitter antenna gain, $G_{R}$ is the receiver antenna gain, $\lambda$ is the wavelength, $d$ is the distance between the transmitter and receiver, $A_{\mathrm{cs}}$ is the clear sky attenuation, and $L$ is the system loss factor. Next, define the attenuation factor $a=P_{R} / P_{T}=K^{2} / d^{2}$, where

$$
K=\sqrt{\frac{G_{T} G_{R} A_{\mathrm{cs}} \lambda^{2}}{\left(4 \pi^{2}\right)^{2} L}} .
$$

The cumulative distribution function is given by

$$
\begin{aligned}
\operatorname{Pr}[a \leq A] & =1-\operatorname{Pr}[a>A] \\
& =1-\left(\frac{(1 / 4) \pi\left[K^{2} / A-R_{\min }^{2}\right]}{(1 / 4) \pi\left[R^{2}-R_{\min }^{2}\right]}\right) \\
& =\left(\frac{R^{2}-K^{2} / A}{R^{2}-R_{\min }^{2}}\right) \\
& =F(A) .
\end{aligned}
$$

The pdf of $a$ can be easily obtained by differentiating (16) with respect to $A[20]$ :

$$
p_{A}(a)=\left(\frac{K^{2}}{R^{2}-R_{\min }^{2}}\right) \frac{1}{a^{2}}, \quad \frac{K^{2}}{R^{2}} \leq a \leq \frac{K^{2}}{R_{\min }^{2}} .
$$

From (13), we can write the expression for the pairwise error probability as

$$
P_{2}\left(\mathbf{s}_{l}, \hat{s}_{l}\right) \leq \prod_{k=1}^{l} E_{a} \exp \left(-\frac{a^{2}}{4 N_{0}}\left|s_{k}-\hat{s}_{k}\right|^{2}\right)=\prod_{k=1}^{l} C\left(s_{k}, \hat{s}_{k}\right) .
$$

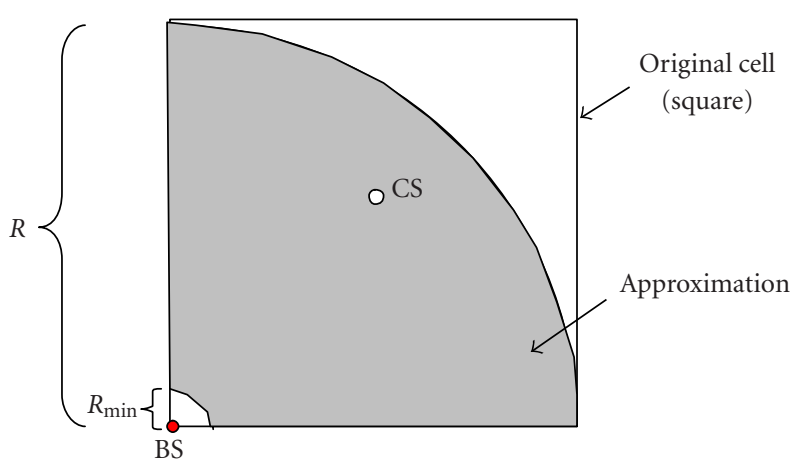

Figure 6: Approximation of the square LMDS cell.

The Chernoff factor, $C\left(s_{k}, \hat{s}_{k}\right)$, can be written as

$$
C\left(s_{k}, \hat{s}_{k}\right)=\int_{K^{2} / R_{\min }^{2}}^{K^{2} / R^{2}} p_{A}(a) \exp \left(-\frac{a^{2}}{4 N_{0}}\left|s_{k}-\hat{s}_{k}\right|^{2}\right) d a .
$$

Combining (17) and (19) and simplifying, we obtain

$$
\begin{gathered}
C\left(s_{k}, \hat{s}_{k}\right) \\
=\int_{K^{2} / R_{\min }^{2}}^{K^{2} / R^{2}}\left(\frac{K^{2}}{R^{2}-R_{\min }^{2}}\right) \frac{1}{a^{2}} e^{-\delta_{k}^{2} a^{2}} d a \\
=\left[( \frac { K ^ { 2 } } { R ^ { 2 } - R _ { \operatorname { m i n } } ^ { 2 } } ) \left(-a^{2} e^{-\delta_{k}^{2} a^{2}}-\delta_{k}^{2} \sqrt{\pi}\right.\right. \\
\left.\left.\cdot \operatorname{erf}\left(\delta_{k}^{2} a^{2}\right)\right)\right]_{K^{2} / R^{2}}^{K^{2} / R_{\min }^{2}} \\
=\left[( \frac { K ^ { 2 } } { R ^ { 2 } - R _ { \operatorname { m i n } } ^ { 2 } } ) \left(\frac{1}{K^{2}}\left(R^{2} e^{-\delta_{k}^{2}\left(K^{4} / R^{4}\right)}-R_{\min }^{2} e^{-\delta_{k}^{2}\left(K^{4} / R_{\min }^{4}\right)}\right)\right.\right. \\
-\delta_{k}^{2} \sqrt{\pi} \cdot\left(\operatorname{erf}\left(\delta_{k}^{2} \frac{K^{2}}{R^{2}}\right)\right. \\
\left.\left.\left.\quad-\operatorname{erf}\left(\delta_{k}^{2} \frac{K^{2}}{R_{\min }^{2}}\right)\right)\right)\right],
\end{gathered}
$$

where $\delta_{k}^{2}=\left|s_{k}-\hat{s}_{k}\right|^{2} / 4 N_{0}$. From (18) and (20), the pairwise error probability is given by

$$
\begin{aligned}
& P_{2}\left(\mathbf{s}_{l}, \hat{\mathbf{s}}_{l}\right) \leq \prod_{k=1}^{l}\left[\left(\frac{K^{2}}{R^{2}-R_{\min }^{2}}\right)\right. \\
& \times\left(\frac{1}{K^{2}}\left(R^{2} e^{-\delta_{k}^{2}\left(K^{4} / R^{4}\right)}-R_{\min }^{2} e^{-\delta_{k}^{2}\left(K^{4} / R_{\min }^{4}\right)}\right)\right. \\
&\left.\left.\quad-\delta_{k}^{2} \sqrt{\pi} \cdot\left(\operatorname{erf}\left(\delta_{k}^{2} \frac{K^{2}}{R^{2}}\right)-\operatorname{erf}\left(\delta_{k}^{2} \frac{K^{2}}{R_{\min }^{2}}\right)\right)\right)\right] .
\end{aligned}
$$

We now consider the low SNR and the high SNR cases separately. 


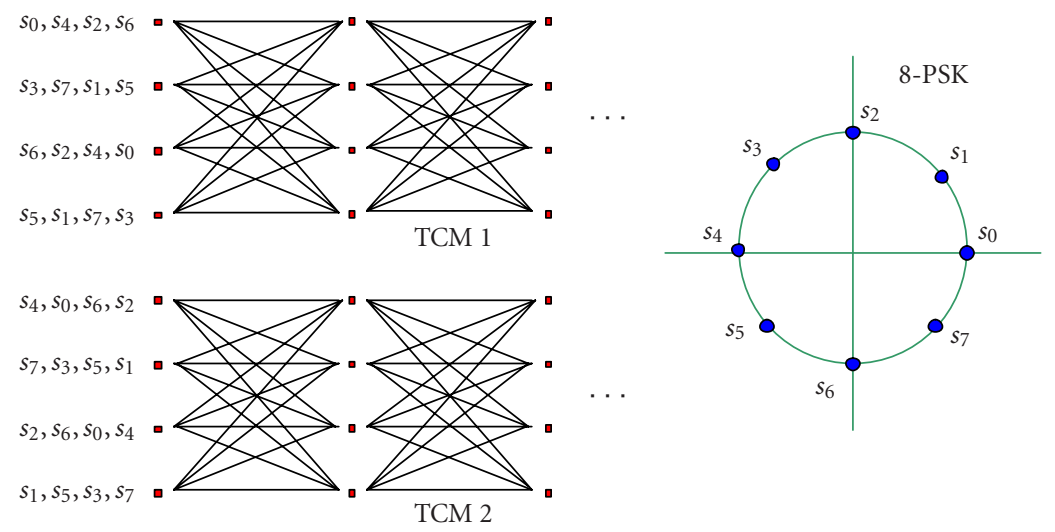

Figure 7: The two TCM schemes employed in the cochannel cells.

\section{Pairwise error probability for low SNR}

For the low SNR scenario, we assume $\delta_{k}^{2}\left(K^{2} / R_{\min }^{2}\right) \ll 1$. It may be recalled that $\delta_{k}^{2}$ is a measure of the SNR and $\left(K^{2} / R_{\min }^{2}\right)$ represents the maximum attenuation value (i.e., the largest possible value of $a_{i}$ ) for the desired signal. Using the approximations $e^{-x} \approx 1-x$ and $\operatorname{erf}(x) \approx 2 x / \sqrt{\pi}$ for $x \ll 1$, equation (21) can be simplified to

$$
P_{2}\left(\mathbf{s}_{l}, \hat{\mathbf{s}}_{l}\right) \leq \prod_{k=1}^{l}\left(1-\frac{K^{2}}{R_{\min }^{2}} \frac{K^{2}}{R^{2}} \frac{\left|s_{k}-\hat{s}_{k}\right|^{2}}{4 N_{0}}\right) .
$$

If we represent the maximum attenuation value within the cell by $a_{\max }=\left(K^{2} / R_{\min }^{2}\right)$ and the minimum attenuation value within the cell by $a_{\min }=\left(K^{2} / R^{2}\right)$, and use the approximation $\prod_{k}\left(1-x_{k}\right) \approx 1-\sum_{k} x_{k}$ for $x \ll 1$, (22) can be written as

$$
\begin{aligned}
P_{2}\left(\mathbf{s}_{l}, \hat{\mathbf{s}}_{l}\right) & \leq 1-\sum_{k=1}^{l} \frac{K^{2}}{R_{\min }^{2}} \frac{K^{2}}{R^{2}} \frac{\left|s_{k}-\hat{s}_{k}\right|^{2}}{4 N_{0}} \\
& =1-\left(\frac{a_{\max } a_{\min }}{4 N_{0}}\right) d_{E}^{2}\left(l_{\mathrm{eff}}\right),
\end{aligned}
$$

where the sum of the squared Euclidean distance, $d_{E}^{2}\left(l_{\text {eff }}\right)=$ $\sum_{k=1, s_{k} \neq \hat{s}_{k}}^{l}\left|s_{k}-\hat{s}_{k}\right|^{2}$ and $l_{\text {eff }}$ is the effective length of the error event. Thus, the design rule for the low SNR scenario is to maximize the minimum $d_{E}^{2}\left(l_{\text {eff }}\right)$.

\section{Pairwise error probability for high SNR}

For the high SNR case, we assume $\delta_{k}^{2}\left(K^{2} / R^{2}\right) \gg 1$. Using the assumption that $\operatorname{erf}(x) \approx 1-\left(e^{-x^{2}} / \sqrt{\pi} x\right)\left(1-1 / 2 x^{2}\right)$ for $x \gg 1$ and neglecting $R_{\min }^{2} e^{-\delta_{k}^{2}\left(K^{4} / R_{\min }^{2}\right)}$ with respect to $R^{2} e^{-\delta_{k}^{2}\left(K^{4} / R^{4}\right)}$, equation (21) can be simplified to

$$
\begin{aligned}
P_{2}\left(\mathbf{s}_{l}, \hat{\mathbf{s}}_{l}\right) \leq & \prod_{k=1}^{l} \frac{1}{2}\left(\frac{R^{2}}{R^{2}-R_{\min }^{2}}\right)\left(\frac{4 N_{0}}{\left|s_{k}-\hat{s}_{k}\right|^{2}}\right) \\
& \times\left(\frac{1}{a_{\min }^{2}}\right) \exp \left\{-\frac{\left|s_{k}-\hat{s}_{k}\right|^{2}}{4 N_{0}} a_{\min }^{2}\right\} .
\end{aligned}
$$

Defining the product of the squared Euclidean distance, $d_{P}^{2}\left(l_{\text {eff }}\right)=\prod_{k=1, s_{k} \neq \hat{s}_{k}}^{l}\left|s_{k}-\hat{s}_{k}\right|^{2}$ for the effective length, $l_{\text {eff }}$, equation (24) can be written as

$$
\begin{aligned}
P_{2}\left(\mathbf{s}_{l}, \hat{\mathbf{s}}_{l}\right) \leq & \left(\frac{R^{2}}{R^{2}-R_{\min }^{2}}\right)^{l_{\mathrm{eff}}}\left(\frac{2 N_{0}}{a_{\min }^{2}}\right)^{l_{\mathrm{eff}}} \\
& \times \frac{1}{d_{P}^{2}\left(l_{\mathrm{eff}}\right)} \exp \left\{-\frac{a_{\min }^{2}}{4 N_{0}} d_{E}^{2}\left(l_{\mathrm{eff}}\right)\right\} .
\end{aligned}
$$

Thus, the design rule for the high SNR scenario is to maximize both the minimum $d_{E}^{2}\left(l_{\mathrm{eff}}\right)$ and the minimum $d_{P}^{2}\left(l_{\mathrm{eff}}\right)$.

\section{NUMERICAL SIMULATIONS}

In this section we discuss the proposed TCM schemes and the results obtained by numerical simulations. We propose a 4-state, fully connected trellis followed by a mapper. The TCM schemes for the cochannel cells are shown in Figure 7. The labels of the branches are written on the left. For example, the branch connecting node 1 to node 1 is labeled with $s_{0}$, the branch connecting node 1 to node 2 is labeled with $s_{4}$, and so forth, where $s_{i}, i=0,1, \ldots, 7$, are the symbols from the 8-PSK constellation diagram. This encoder takes in 2 bits at a time and adds an additional bit. Thus the rate of this encoder is $2 / 3$. In order to achieve the same data rate, we have to pay an additional bandwidth penalty of $3 / 2$ (since only $2 / 3$ of the bandwidth is used to send information, the rest $1 / 3$ is overhead). Since the input is 2 bits at a time, every node of the trellis has $2^{2}=4$ outgoing branches. A sequence of incoming bits is translated into a sequence of outgoing symbols. At the decoder, Viterbi decoding is performed to recover the transmitted symbols. For both these TCM schemes, $d_{E}^{2}\left(l_{\text {eff }}\right)=3.712 E_{s}$ and $d_{P}^{2}\left(l_{\text {eff }}\right)=4.688 E_{s}^{2}$, where $E_{s}$ represents the average energy per symbol for the digital modulation [21].

Simulations have been carried out to test the performance of the system using one-frequency, two-polarization, and two TCM schemes. The design criterion for the two TCM schemes (given in Figure 7) is to maximize the minimum $d_{E}^{2}\left(l_{\text {eff }}\right)$. This is the common design criterion for both the low and high SNR scenarios, as derived in Section 4. By using these 8-PSK/TCM it was found by simulations that the areas within the cell where $\mathrm{C} / \mathrm{I}$ exceeded $8 \mathrm{~dB}, P_{\text {sym }}$ was less than $10^{-6}$. This implies that all regions where $\mathrm{C} / \mathrm{I}>8 \mathrm{~dB}$, 
acceptable QoS will be available if we deploy the two TCM schemes in the cochannel cells. From Table 1, we note that only $3.77 \%$ of the total cell area has $\mathrm{C} / \mathrm{I}<8 \mathrm{~dB}$ for the $1 \mathrm{~F} 2 \mathrm{P} 2 \mathrm{~T}$ case. Thus, only $3.77 \%$ of the whole cell will have unacceptable QoS. Comparing this with the pure 8-PSK case using 2F2P, we find that acceptable QoS means C/I > 9.5 dB. This corresponds to $5.48 \%$ of the cell area having poor QoS. Thus, the 1F2P2T scheme outperforms the 2F2P scheme. The improvement in performance is due to the coding gain. The signal from the interfering cell is being treated like "noise" by TCM scheme 1 and hence rejected. The asymptotic coding gain [10] of TCM 1 and TCM 2 with respect to uncoded 8 -PSK is given by

$$
g_{\infty}=10 \log _{10}\left(\frac{d_{\text {free }}^{2}}{d_{8-\mathrm{PSK}}^{2}}\right)=10 \log _{10}\left(\frac{3.712}{0.586}\right)=8.017 \mathrm{~dB}
$$

We next look at the reduction in the bandwidth requirement offered by the 1F2P2T scheme. Our TCM scheme takes 2input bits and outputs 3 bits, which are then mapped on to one of the possible 8 symbols of 8 -PSK. The code rate of our encoder is $2 / 3$. This means that $2 / 3$ of the total bandwidth will be used for data transmission and the remaining $1 / 3$ of the bandwidth will be used for coding overhead. Hence, for this $2 / 3$ rate TCM scheme, the net bandwidth required will be $(1 / 2) /(2 / 3)=3 / 4$ of the original bandwidth, providing a bandwidth reduction of $25 \%$. The factor of $1 / 2$ is because we are using just one frequency instead of two. In general, for a rate $(m /(m+1))$ TCM encoder, the bandwidth reduction is given by

$$
\begin{aligned}
\operatorname{BW} \text { reduction }(\%) & =100\left(1-\frac{1}{2} \frac{(m+1)}{m}\right) \\
& =\frac{100(m-1)}{2 m} .
\end{aligned}
$$

This suggests that the code rate of the trellises should be greater than $1 / 2$ in order to cause a reduction in the effective bandwidth used. The plot of the percentage bandwidth reduction versus $m$ is given in Figure 8 . The asymptotic value of bandwidth reduction is $50 \%$. From the figure it is clear that $40 \%$ bandwidth reduction is achieved simply by using a $5 / 6$ encoder. It should be noted that a larger value of $m$ implies a higher decoder complexity.

\section{Tradeoffs between the proposed and the existing schemes}

We next discuss the trade-offs between the two strategies: two-frequency, two-polarization $(2 \mathrm{~F} 2 \mathrm{P})$ and the one-frequency, two-polarization, and two-TCM schemes (1F2P2T). The trade-offs are discussed under the heads of bandwidth requirement, complexity, and frequency planning.

(1) Bandwidth requirement: there is always a reduction in the bandwidth used when we use the 1F2P2T as opposed to the 2F2P scheme. From equation (27) we see that for a rate $(m /(m+1))$ TCM encoder, the achieved bandwidth reduction is $100(m-1) / 2 m \%$. The asymptotic value of bandwidth reduction is $50 \%$. From Figure 8 it is clear that $40 \%$ bandwidth reduction

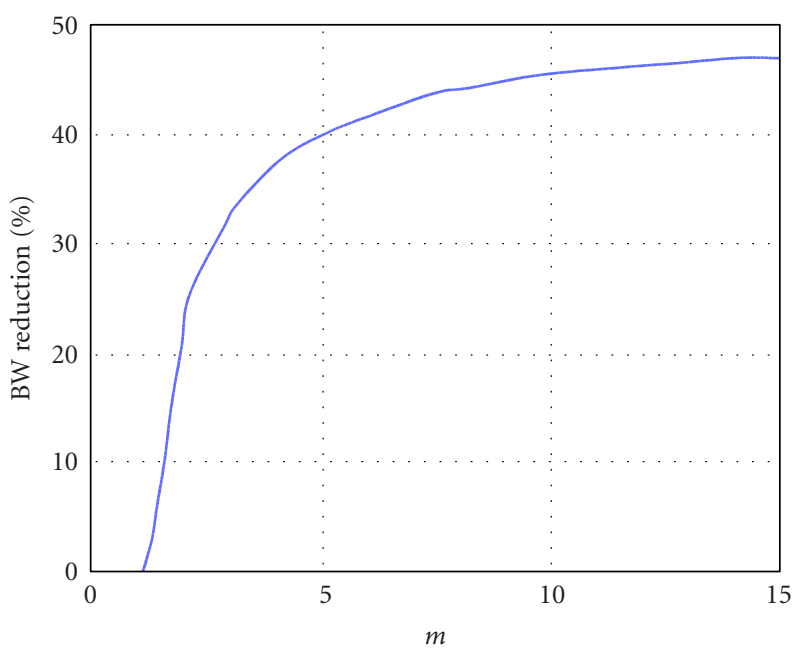

FIGURE 8: Bandwidth reduction with increasing $m$, where $(m / m+1)$ is the rate of the encoder.

is achieved simply by using a rate $5 / 6$ encoder. A higher rate encoder can also provide a better coding gain and thus a better performance. We also observe the law of diminishing returns as we increase the value of $m$.

(2) Complexity: by introducing two TCM schemes, we have added computational complexity to the system. However, very fast TCM encoders and decoders are available off-the-shelf. Hence, the additional complexity will not necessarily translate into additional costs.

(3) Frequency planning: the $2 \mathrm{~F} 2 \mathrm{P}$ scheme requires a more complex frequency planning than the 1F2P2T scheme, since there is only one frequency to layout in the latter scheme. The coding diversity (different TCM encoders) will have to be enabled at the BS transmitters.

We have only considered the downlink in this paper. The treatment of the uplink scenario is similar when power control is not used [15]. For the uplink, the interfering customer stations (ICS) located in three different interfering cells IC 1, IC 2, and IC 3 contribute to the CCI at the BS facing interference (BSI). If we consider only the azimuth radiation pattern of the transmitting antennas of the customer sites, the number of ICS within these three sectors of IC 1 to IC 3 in the uplink are the same as the number of interfered customers within the three sectors of the desired BS cell in the downlink, on an average. However, in contrast to the downlink, where a customer receives only one interfering signal from one of the three interfering base stations (IBSs) due to the narrow HPBW of the CS, it is possible, that the BS under consideration (BSI) in the uplink receives at the same time as three interfering signals with the same frequency and time slot from one customer in each of the three interfering cells IC 1 to IC 3 . Hence, in case of three accumulating signals, the resulting interference level could be at most $4.8 \mathrm{~dB}$ higher than for a single interfering signal. This would be the case if in each interfering cell (IC) there is a CS with LOS to the base station interfered (BSI) transmitting with the same frequency and time slot at the same time. 


\section{CONCLUSIONS}

In this paper we propose a new scheme to increase the capacity of interference-limited LMDS networks. We suggest the use of two different TCM schemes in cochannel cells in order to mitigate the effect of interference. It is shown that the frequency reuse of unity is possible using this scheme. Thus we can use one-frequency, two-polarization, and two TCM codes (1F2P2T) for LMDS networks. LOS downlink scenario has been considered. Analytical expressions for pairwise error probability have been worked out for the case when channel state information is available. The design rules are as follows: (i) for the low SNR scenario, maximize the minimum squared Euclidean distance, $d_{E}^{2}\left(l_{\text {eff }}\right)$ and (ii) for the high SNR scenario, maximize both the minimum squared Euclidean distance, $d_{E}^{2}\left(l_{\mathrm{eff}}\right)$, and the minimum squared product distance, $d_{P}^{2}\left(l_{\text {eff }}\right)$.

Numerical simulations have been performed to test out the proposed scheme $(1 \mathrm{~F} 2 \mathrm{P} 2 \mathrm{~T})$. It has been found that for the scenario that uses 8 -PSK/TCM, a reduction in the bandwidth requirement of about $25 \%$ is possible. This can translate into increased capacity, or better QoS to preferred customers. The relative trade-offs between the existing scheme and the proposed scheme are also discussed. It is also shown that by using TCM in interference-limited LMDS network, one can increase the capacity of the network by $50 \%$.

\section{ACKNOWLEDGMENT}

The author wishes to thank Professor R. Jakoby of the Wireless Communication Lab, Technical University of Darmstadt, Germany, for the initial discussions on this topic.

\section{REFERENCES}

[1] H. Petry, "Broadband wireless access-where are we today?" in Proc. Mikrowellen und Optonik/Microwaves and Optronics (MIOP '01), Stuttgart, Germany, May 2001.

[2] P. B. Papazian, G. A. Hufford, R. J. Achatz, and R. Hoffman, "Study of the local multipoint distribution service radio channel," IEEE Transactions on Broadcasting, vol. 43, no. 2, pp. 175-184, 1997.

[3] S. Q. Gong and D. Falconer, "Cochannel interference in cellular fixed broadband access systems with directional antennas," Wireless Personal Communications, vol. 10, no. 1, pp. 103-117, 1999.

[4] R. Bultitude, P. Hou, R. Hahn, G. Hendrantoro, D. Falconer, and R. Berube, "Radio propagation data pertinent to the design of LMCS systems at $28 \mathrm{GHz}$," in Proc. Symposium on Antenna Technology and Applied Electromagnetics (ANTEM '98), Ottawa, Canada, August 1998.

[5] D. A. Gray, "A broadband wireless access system at $28 \mathrm{GHz}$," in NIST-Cosponsored Wireless Communications Conference, pp. 1-7, Boulder, Colo, USA, August 1997.

[6] A. Seville, M. Willis, and E. Falaise, "Area coverage studies for millimetre-wave services," in Proc. Millennium Conference on Antenna and Propagation, Davos, Switzerland, April 2000.

[7] A. F. Elrefaie and M. Shakouri, "Propagation measurements at $28 \mathrm{GHz}$ for coverage evaluation of local multipoint distribution service," in Proc. Wireless Communications Conference, pp. 12-17, Boulder, Colo, USA, August 1997.
[8] ACTS Project, "Cellular radio access for broadband services (CRABS)," AC215, Telenor R\&D, Kjeller, Norway, February, 1999.

[9] R. Bose and A. Kumar, "Reducing frequency reuse distance in cellular communications using trellis coded modulation," IEE Proceedings-Communications, vol. 148, no. 3, pp. 149-153, 2001.

[10] S. H. Jamali, Coded-Modulation Techniques for Fading Channels, vol. 268 of The Kluwer International Series in Engineering and Computer Science, Springer, New York, NY, USA, 1994.

[11] E. Biglieri, D. Divsalar, P. J. McLane, and M. K. Simon, Introduction to Trellis-Coded Modulation with Applications, Macmillan, New York, NY, USA, 1991.

[12] ITU-R, "Prediction procedure for the evaluation of microwave interferrence between stations on the surface of the earth at frequencies above $0.7 \mathrm{GHz}$," ITU-R Recommendation 452-6, PN Series, Propagation in Non Ionized Media, pp. 565$591,1990$.

[13] ITU-R, "Propagation data and prediction methods required for the design of terrestrial line-of-sight systems," ITU-R Recommendation P.530-9, 2001.

[14] R. Jakoby and M. Grigat, "MMDS zur Erweiterung von BK-Netzen," Research report from the Research Center of Deutsche Telekom AG Darmstadt, 1996.

[15] R. Bose, G. Bauer, and R. Jakoby, "Two-dimensional line of sight interference analysis of LMDS networks for the downlink and uplink," IEEE Transactions on Antennas and Propagation, vol. 52, no. 9, pp. 2464-2473, 2004.

[16] R. Bose, A. Hayn, and R. Jakoby, "Intra- and inter cell interference investigations for broadband radio access systems above $10 \mathrm{GHz}$," Journal of Telecommunications and Information Technology, no. 2, pp. 10-20, June 2001.

[17] A. Hayn, R. Bose, and R. Jakoby, "Multipath propagation and LOS interference studies for LMDS architecture," in Eleventh International Conference on Antennas and Propagation (ICAP '01), pp. 686-690, Manchester, UK, April 2001.

[18] J. G. Proakis, Digital Communications, McGraw-Hill, New York, NY, USA, 1983.

[19] W. C. Y. Lee, Mobile Communications Engineering, McGrawHill, New York, NY, USA, 1982.

[20] A. Papoulis, Probability, Random Variables and Stochastic Processes, McGraw-Hill, New York, NY, USA, 3rd edition, 1991.

[21] R. Bose, Information Theory, Coding and Cryptography, McGraw-Hill, Singapore, 2003.

Ranjan Bose received his B.Tech. degree in electrical engineering from the Indian Institute of Technology (IIT) Kanpur, in 1992, and the M.S. and Ph.D. degrees in electrical engineering from the University of Pennsylvania, Philadelphia, USA, in 1993 and 1995, respectively. He worked at Alliance Semiconductor Inc., San Jose, as a Senior Design Engineer from 1996 to 1997. Since November 1997, he has been with the Department of Electrical Engineering, IIT Delhi, where currently he is an Associate Professor. His research interests lie in the areas of broadband wireless access and coding theory. Dr. Bose received the URSI Young Scientist Award in 1999, the Humboldt Fellowship in July 2000, and the Indian National Academy of Engineers (INAE) Young Engineers Award in 2003. 